\title{
Placental regulation of fetal nutrient supply
}

\author{
Elvira Larquéa , María Ruiz-Palacios ${ }^{\mathrm{a}}$, and Berthold Koletzko ${ }^{\mathrm{b}}$
}

\begin{abstract}
Purpose of review
Placental nutrient uptake and transfer may have a unique role, as changes in trophoblast nutrient-sensing signaling pathways regulate cell metabolism and may affect the fetal growth and health programming in the offspring.
\end{abstract}

\section{Recent findings}

The functionality of the placenta could affect the neonatal adiposity and the fetal levels of key nutrients such as long-chain polyunsaturated fatty acids. Insulin, oxygen and amino acid concentrations may regulate the mammalian target of rapamycin (mTOR) nutrient sensor in the human placenta affecting trophoblast metabolism and nutrient delivery.

\begin{abstract}
Summary
The mechanisms involved in both placental uptake and transfer of macronutrients are reviewed. Fatty acid, cholesterol and amino acid transport across the placenta involves a complex system to ensure nutrient delivery to the growing fetus. The placental glucose transfer is important for fetal macrosomia, but lipid disturbances in both maternal and placental compartments may contribute to neonatal fat accretion. Maternal insulin has little effect on the avidity of glucose transport by the placenta, but may interfere in placental metabolism via mTOR nutrient sensor. mTOR is a positive regulator of the amino acid carriers and constitutes a critical link between maternal nutrient availability and fetal growth, thereby influencing the long-term health of the fetus.
\end{abstract}

\section{Keywords}

amino acids, cholesterol, fatty acids, glucose, nutrient sensor, placenta

\section{INTRODUCTION}

The intrauterine development of the fetus depends on an adequate nutrient supply via the placenta. This is not achieved by simple diffusion of all substrates, but the transfer of some nutrients is regulated by the placenta itself. Some nutrients and substrates are metabolized by the placenta, and the placental expression of specific transport proteins appears to be adapted to fetal demands. The regulation of placental enzymes, receptors and transport proteins will determine the extent of glucose, lipids and amino acid transfer to the fetus that strongly contributes to fetal growth.

\section{MECHANISIMS INVOLVED IN THE PLACENTAL GLUCOSE TRANSFER}

Glucose is the main source of energy for the fetus, although lipids also play an important role. In pregnant women infused with glucose labeled with stable isotopes some hours before delivery, it was shown that over 95\% of fetal plasma glucose was derived from maternal plasma [1].
The placental glucose transport occurs by facilitated diffusion mediated mainly by the glucose carrier GLUT-1, which is a carrier independent of insulin. GLUT-1 has been identified in the placental trophoblast cells with an asymmetrical distribution, being more abundant in the microvillous membrane (maternal face) in contrast to the basal membrane (fetal face). This asymmetrical distribution has been proposed as a rate-limiting step in the transplacental glucose transfer from the mother to the fetus [2]. In gestational diabetes mellitus (GDM), enhanced levels of GLUT-1 in the basal membrane of the placenta were reported which could lead to a higher transfer of glucose to the fetal circulation.

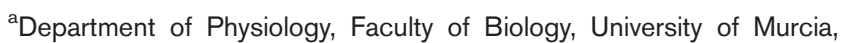
Murcia, Spain and ${ }^{b}$ Dr von Haunersches Kinderspital, Ludwig-Maximilians University, Munich, Germany

Correspondence to Elvira Larqué, Department of Physiology, Faculty of Biology, University of Murcia, Campus Espinardo 30100, Murcia, Spain. Tel: +34 868884239; fax: +34 868883963; e-mail: elvirada@um.es
}

Curr Opin Clin Nutr Metab Care 2013, 16:292-297

DOI:10.1097/MCO.0b013e32835e3674 


\section{KEY POINTS}

- Glucose and lipid metabolism may interfere with amino acid transport through nutrient signal pathways.

- Placental tissue uptakes preferentially long-chain polyunsaturated fatty acids (LC-PUFAs) with respect to other fatty acids, but the mechanisms involved in this preferential transfer should be clarified in healthy and pathological pregnancies.

- There is a competition for placental cholesterol uptake from the maternal lipoproteins by different carriers, but the relevance of each cholesterol carrier in the whole materno-fetal transfer process is yet unknown.

However, within the physiological range, GLUT-1 expression is relatively refractory to glucose concentration. Moreover, GLUT-3 is present in the endothelial walls of umbilical arteries and recently it has been described also in placental syncytial microvillous membrane [3]. GLUT-3 shows high expression in placenta early in gestation, decreasing at the end of pregnancy [4]. In fact, GLUT-3 mutations cause early pregnancy loss and fetal growth restriction [5]. The different expression of GLUT-3 in placenta along gestation has been proposed to be associated with the local changes of CRH synthesis by the placenta [3], although other mechanisms could be also implicated. Interestingly, brain and placental tissue both are dependent upon these two glucose transporter (GLUT) isoforms GLUT-1 and GLUT-3 for glucose uptake, which are not insulin responsive.

The role of GLUT-4 in the placental glucose transfer is uncertain. GLUT-4 (which is the main glucose carrier in the organism and that depends on insulin) has only been identified in the stroma of the placental villous, but not in syncytiotrophoblast cells. Recently, some fructose-specific transporters like GLUT-9 and GLUT-11 or even GLUT-10 and GLUT-12 have also been identified in placental tissue, but their role in regard to glucose transport remains uncertain [6]. Epigenetic studies indicate that DNA methylation regulates the expression of GLUT-3 and GLUT-10 during gestation, whereas the constitutively expressed GLUT-1 showed no promoter methylation [7].

Insulin and protein hormones do not cross placental tissue. Maternal insulin has little effect on the avidity of glucose transport by the placenta, but interfere modifying glucose levels in maternal circulation [8]. The role of insulin in the placental glucose uptake is very limited, as syncytiotrophoblast do not express GLUT-4 carriers. Nevertheless, the activation of the insulin receptor in the placenta

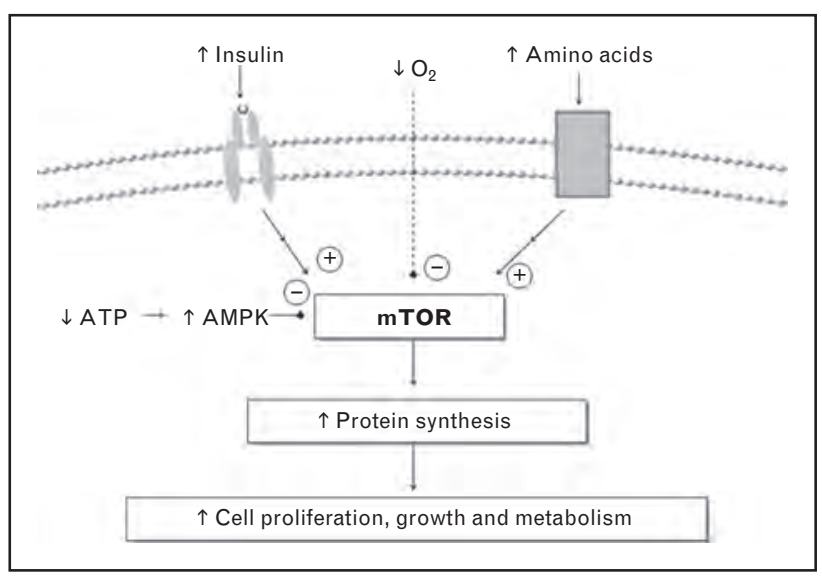

FIGURE 1. Signaling mechanisms of mTOR and link between maternal nutrient availability and fetal growth.

upregulate the nutrient sensor signal, mammalian target of rapamycin (mTOR) [9"]. mTOR is a positive regulator of placental amino acid transporters and stimulates cell proliferation and growth, promoting an anabolic metabolic phenotype Fig. 1 (mTOR signaling). Preliminary studies indicate an activation of placental mTORC1 signaling in association with maternal obesity $\left[10^{*}\right]$ and probably with GDM, which could link insulin levels in these patients. This could explain in part the higher size of GDM placentas with respect to the controls classically described. Nevertheless, there is also a downregulation of the insulin signal pathway by insulin receptor internalization via Annexin A2 in GDM placental villi, leading to insulin resistance even in GDM placentas [11].

The placenta can also partially regulate its glucose level by the synthesis of glycogen. Although some gluconeogenic enzymes were known to be present, the placenta was previously considered incapable of glucose secretion because it lacked glucose-6-phosphatase. However, further studies have demonstrated that specific glucose-6-phosphatase is present in the placenta, but it may be the product of a different gene from conventional hepatic glucose-6-phosphatase. GDM placentas have abundant depots of glycogen in its structure pointing to another mechanism of glucose transfer modulation [12].

Fetal macrosomia and its complications may occur even in adequately controlled diabetic mothers, suggesting that maternal glycemia is not the only determinant of fetal glycemic status and well-being. Both maternal triglycerides and nonesterified fatty acids (NEFAs) levels have been shown to be correlated with both neonatal weight and fat mass in GDM, indicating that maternal hyperlipidemia in GDM actively enhances the availability of lipids to the fetus [13]. The underlying mechanisms 
by which maternal hyperglycemia regulates placental lipid metabolism and translate into fetal adiposity are incompletely understood.

\section{MECHANISMS INVOLVED IN THE}

PLACENTAL TRANSFER OF FATTY ACIDS

There is a relatively high placental transfer of lipids in humans, and even the fetus may synthesize some saturated fatty acids and monounsaturated fatty acids from glucose. Lipids are transferred because the fetus requires essential linoleic and $\alpha$-linolenic acid, and additionally long-chain polyunsaturated fatty acids (LC-PUFAs) to match the in-utero accretion rate especially during the brain growth spurt.

It has been demonstrated in pregnant women in vivo, using labeled fatty acids with stable isotopes, the preferential placental uptake and transfer of LC-PUFAs from the mother to the fetus with respect to other fatty acids, and specially for docosahexaenoic acid (DHA; n-3 LC-PUFA) [14,15]. This selective LC-PUFA transfer highlights the relevance of the placenta for the fatty acid transfer. In addition, maternal and offspring fatty acid desaturase (FADS) polymorphisms may contribute to determine cord blood n-6 LC-PUFAs, whereas the association with n-3 LC-PUFAs is weaker [16]. It cannot be discarded that fetus could synthesize additional DHA by itself, which might be necessary to satisfy the high fetal demand of DHA during pregnancy.

The mechanisms involved in the placental uptake and transfer of fatty acids were recently reviewed by our group [17"']. In the placenta, two lipases have been until now identified for major fatty acid release from the maternal circulating lipoproteins: lipoprotein lipase (LPL) and endothelial lipase. LPL shows triglyceride lipase activity, whereas endothelial lipase is a phospholipase with little triacylglycerol lipase activity. At the end of pregnancy, endothelial lipase continues to be expressed, whereas LPL is virtually absent in the trophoblast. In addition, maternal circulating NEFAs can be directly uptaken from the placenta.

Numerous membrane-bound proteins are expressed in the trophoblast: fatty acid binding protein plasma membrane (FABPpm), placental plasma membrane fatty acid-binding protein (p-FABPpm), fatty acid translocase (FAT/CD36) and fatty acid transport proteins (FATP-1 to FATP-6) [17"]. Only p-FABPpm seems to be exclusive to placental tissue [18], but this protein has not been genetically sequenced and more evidences on the function and identification of this protein are needed. Once in the cytosol, fatty acids are bound to cytosolic fatty acid binding proteins (FABPs) to interact with subcellular organelles, including the endoplasmic reticulum, mitochondria, lipid droplets and peroxisomes. FABPs are also likely to function in the nucleus through the delivery of specific ligands to nuclear transcription factors, such as the peroxisome proliferator-activated receptors (PPARs) [17"'].

Several studies suggest that the placental uptake of maternal fatty acid is altered by maternal overnutrition. Analysis of human placental lipids has shown that the content of triglycerides is higher in placentas from diabetic pregnancies [19]. Nevertheless, available results on the placental LPL and endothelial lipase levels in GDM patients are inconclusive, maybe because of the low number of patients in such studies; no changes [20,21], increases in endothelial lipase but not in LPL [19], increases in LPL [22"] and even reduction in placental LPL [23], to counteract excessive placental uptake of fatty acids in GDM was reported. Activation of several genes involved in the placental lipid biosynthetic pathways was also reported in the placenta GDM [23], although a decrease in the fatty acid oxidation rate could be also responsible for the enhanced fat store in the placental lipid droplets of GDM.

The relationship between fatty acid carriers and the placental fat storage on GDM is complex because of the numerous fatty acid carriers implicated. Increases in placental membrane carriers FAT/ CD36 and decreases in FATP-4, H-FABP and L-FABP carriers were reported in GDM placenta [22"]. Fatty acid incorporation in placental lipid droplets may also interfere in their rate of fetal transfer. In fact, in GDM, a significant discrepancy between normal LC-PUFA contents in erythrocyte and plasma phospholipids of the mother but reduced LC-PUFA contents in plasma phospholipids of the neonate was found $[24,25]$. These data suggest that LC-PUFA transport from the placenta to the fetal circulation could be impaired in women with gestational diabetes. It is important to discern the reason for the lower levels of DHA in GDM babies in order to identify the best strategy of supplementation with LC-PUFA in the mother, the baby or both.

\section{CHOLESTEROL PLACENTAL TRANSFER}

During the last trimester of pregnancy, maternally derived cholesterol contribute with an average of about $22-40 \%$ to the fetal cholesterol pool [26]. The remaining placental cholesterol, however, is used for sterol synthesis, foremost of progesterone. Maternal hypercholesterolemia may lead to presclerosis lesions already in the fetus which raised the interest for understanding the mechanisms involved in the placental transfer of this cholesterol to design suitable pharmacological treatment in these patients [27]. 


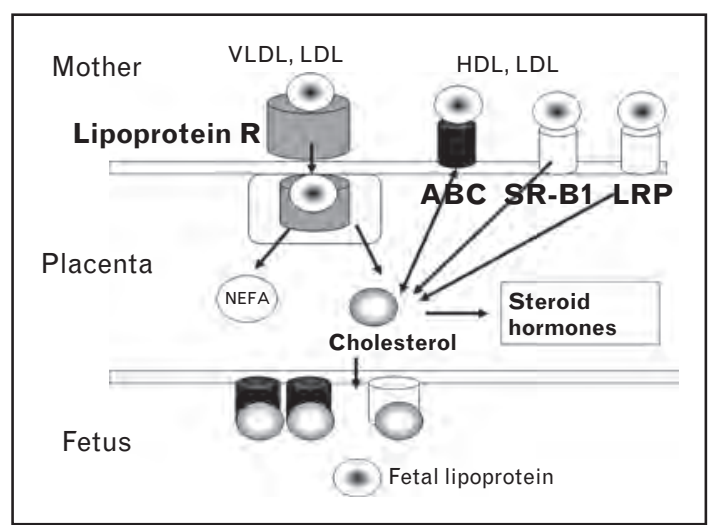

FIGURE 2. Mechanisms involved in the cholesterol transfer across the placenta. Lipoprotein receptor (Lipoprotein R), scavenger receptor class B type 1 (SR-B 1), lipoprotein related protein (LRP), ATP-binding cassette $(A B C)$ transporters $A B C A 1$ and $A B C G 1$, non-esterified fatty acids (NEFA).

Maternal cholesterol can be taken up by the trophoblasts using a lipoprotein-receptor-mediated mechanism (LDL receptor and VLDL-receptor) that requires endocytosis of the particle to lysosomes/ endosomes (Fig. 2); the receptor is recycled to the membrane, whereas the free cholesterol is transported across the cells to metabolically active pools or membranes via Niemann-Pick C1 (NPC1) or other less well defined sterol carrier proteins, like NPC1L1 and SPC- $\mathrm{x} / 2$ (sterol carrier protein) [ $\left.28^{-}\right]$. The endocytosis could also provide fatty acids to the placental tissue by the action of intracellular lipases on the lipoprotein, but the relevance of this mechanism for the placental uptake of fatty acids is still uncertain.

In addition to this transport mechanism, cholesterol uptake is also mediated by other receptors that only transfer the cholesterol across the plasma membrane without internalization of the receptor (Fig. 2). In this way, scavenger receptor class B (SRB1) binds cholesterol mainly from HDL and with less affinity of LDL or modified LDL; LDL-receptor related protein (LRP-1) binds apolipoprotein-E-containing particles such as VLDL. Cubilin and megalin [also named lipoprotein-related protein 2 (LRP-2)] act in concert to bind both HDL and LDL, cubilin binds apo A-1, whereas megalin binds Apo E and Apo B [28"]. ATP-binding cassette (ABC) transporters ABCA1 and ABCG1 mediate the efflux of cholesterol across cellular membranes and removes excess cellular cholesterol [29]. The distribution of $\mathrm{ABC}$ isoforms in apical or basal localization is controversial [30-32]. ABC and SR-B1 seem to be also involved in the cholesterol efflux to fetal lipoproteins $\left[28^{*}, 33\right]$.

Oxysterols formed during cholesterol synthesis as well as by the oxidation of dietary cholesterol may enhance the expression of placental ABCA1 and
ABCG1 carriers, via nuclear liver $X$ receptor (LXR). Treatment of mice with Smith-Lemli-Optiz syndrome (dysfunction on the de novo synthesis of cholesterol) using LXR agonists increases the placental ABCA1 carriers and the materno-fetal cholesterol transfer to the embryos increasing the placental cholesterol efflux [29]. Moreover, low oxygen, like in preeclampsia, increase LXR and ABCA1 carriers which may have consequences for maternal-fetal cholesterol transport [34].

Intrauterine growth restriction (IUGR) is associated with alterations in maternal and fetal lipoproteins. Few studies have evaluated the expression pattern of cholesterol receptors, but the results are controversial. The differential changes in LDL-R and SR-B1 reported in the IUGR placentas warrant further investigations to clarify placental cholesterol uptake in healthy and pathological pregnancies [35].

\section{MECHANISMS INVOLVED IN THE PLACENTAL AMINO ACID TRANSFER}

The size of proteins is the limiting key factor regarding the uptake and transfer of proteins across the placenta. There is no relevant transfer of protein hormones across the placenta, for example, pancreatic or pituitary hormones. Nevertheless, the transfer of free amino acids across the placenta is essential for fetal growth, and the disturbed transfer is associated with IUGR babies. Free amino acid concentration in the placental tissue exceeds by far those of the maternal or fetal circulation which suggest a major role of the placenta in this process.

Amino acids are transferred across the placenta by active transport using a complex system with more than 10 different families of amino acid carriers: systems $\mathrm{A}, \mathrm{L}, \mathrm{y}+, \mathrm{y}+\mathrm{L}, \mathrm{T}, \mathrm{X}_{\mathrm{AG}}^{-}$and ASC (Table 1) [36,37]. These carriers can be classified in two classes: accumulative amino acid transporters (mainly represented by system $\mathrm{A}$ and $\mathrm{X}_{\mathrm{AG}}^{-}$) and amino acid exchangers (LAT1, LAT2, y+LAT1, $\mathrm{y}+\mathrm{LAT} 2$ and ASC) [37]. Accumulative amino acid transporters mediate net uptake of specific amino acids into the syncytiotrophoblast. However, amino acid exchange transporters swap one amino acid within the syncytiotrophoblast for the one in the maternal or fetal circulation, thus altering the composition but not the overall quantity of amino acids transported. Recently, another novel transport system has also been identified, amino acid efflux carriers, that transports amino acids only from the placental cytosol into the fetal circulation [38]. Unlike other transporters, efflux transporters may increase the fetal amino acid concentrations, and the expression of placental LAT3 and TAT1 efflux 
Table 1. Amino acid transport system in the human placenta

\begin{tabular}{|c|c|c|c|c|}
\hline Transport system & Mechanism & Protein & Localization & Substrate \\
\hline \multicolumn{5}{|c|}{$\mathrm{Na}^{+}$-dependent systems } \\
\hline A & $\mathrm{ACC}$ & SNAT1,2,4 & MVM, BM & Neutral amino acids \\
\hline ASC & EX & ASCT1,2 & $\mathrm{BM}$ & Neutral amino acids \\
\hline$\beta$ & & TAUT & MVM, BM & Taurine \\
\hline N & & $\mathrm{SN1}$ & MVM (contested in humans) & Histidine, asparagine, glutamine \\
\hline$X_{A G}^{-}$ & ACC/EX & EAAT1-4 & MVM, BM & Anionic amino acids \\
\hline GLY & & GLYT1 & MVM & Glycine and sarcosine \\
\hline $\mathrm{B}^{\mathrm{O},+}$ & & $\mathrm{ATB}^{\mathrm{O},+}$ & $?$ & Cationic and neutral amino acids \\
\hline \multicolumn{5}{|c|}{$\mathrm{Na}^{+}$-independent systems } \\
\hline L & EX/EF & LAT $1,2,4 / 4 F 2 h c$ & $M V M, B M$ & $\begin{array}{l}\text { Neutral amino acids, branched-chain } \\
\text { amino acids and tryptophan }\end{array}$ \\
\hline$y^{+}$ & Uniporter & CAT1, 4 & MVM, BM & Cationic amino acids \\
\hline$y^{+} L$ & EX & $\mathrm{y}^{+} \mathrm{LAT} 1 / 4 \mathrm{~F} 2 \mathrm{hc}$ & MVM, BM & $\begin{array}{l}\text { Cationic amino acids (neutral amino } \\
\text { acids in the presence of sodium) }\end{array}$ \\
\hline$b^{0,+}$ & EX & rBAT & $B M$ & Cationic and neutral amino acids \\
\hline $\mathrm{T}$ & $\mathrm{EF}$ & TAT1 & $B M$ & Aromatic amino acids \\
\hline Asc & EX & $\mathrm{ascl} / 4 \mathrm{~F} 2 \mathrm{hc}$ & $\mathrm{BM} ?$ & Small neutral amino acids and D-serine \\
\hline
\end{tabular}

ACC, accumulative; BM, basal membrane; EF, efflux; EX, exchange; MVM, microvillous membrane. Data from [36] and [37].

carriers has shown positive associations with the infant size at birth [38]. Moreover, amino acids may also be metabolized within the placenta, and glutamate is converted in glutamine, or even can be transferred through the fetal circulation. Altogether, these observations point to a complex system to facilitate nutrient delivery to warrant the demand of the growing fetus.

Insulin, oxygen and amino acid concentrations may regulate mTOR nutrient sensor in the human placenta (Fig. 1). mTOR is a positive regulator of the amino acid carriers system A and system L [39]. mTOR activity is inhibited in the IUGR placentas consistent with the possibility that the nutrient and oxygen levels are low in the trophoblast (Fig. 1) [40]. On the contrary, it was reported that activation of placental mTOR signaling and amino acid transporters in obese women give birth to large babies [10"]. Maternal adiponectin levels decrease with maternal obesity and are inversely correlated to birth weight; chronic maternal infusion of adiponectin in pregnant mice downregulated the placental amino acid transporters and decreased the fetal growth [41"]. This effect was mediated by the inhibition of insulin/IGF-1 and mTOR signaling pathways linking another novel physiological mechanism by which the endocrine functions of maternal adipose tissue influence fetal growth. In other words, the placenta acts as a 'nutrient sensor' regulating its transporter function.
Amino acid transporters also contribute to the placental transport of other key molecules such as thyroid hormones $\mathrm{T} 3$ and $\mathrm{T} 4$ by a putative system $\mathrm{T}$ and system $\mathrm{L}$ transporters. In addition, systems $\mathrm{L}, \mathrm{A}$ and $\mathrm{y}(+) \mathrm{L}$ are involved in placental homocysteine (Hcy) transport [42]. Elevated maternal plasma levels of Hcy are associated with pregnancy complications and adverse neonatal outcomes, suggesting placental transport of Hcy may impact on fetal development.

\section{CONCLUSION}

Placental tissue is dependent upon GLUT isoforms for glucose uptake that are not insulin responsive. Insulin upregulates mTOR in the placenta and interferes with the amino acid uptake and cell growth. Higher placental lipid droplet accumulation and impaired LC-PUFA transfer was reported in GDM which might adversely affect the offspring. The placental cholesterol is mainly used for steroid hormone synthesis and also for fetal transfer. The relevance of each cholesterol carrier should be clarified. It is possible that the trophoblast mTOR signaling links maternal nutrient availability to fetal growth by modulating the flux of amino acids across the placenta.

\section{Acknowledgements}

Additional support from the Hero Institute for Infant Nutrition, Hero Spain S.A. is gratefully acknowledged. 


\section{Conflicts of interest}

This article does not necessarily reflect the views of the Commission and in no way anticipates the future policy in this area.

Elvira Larque is a recipient of the ESPGHAN Award for Young Investigators. Budapest 2009. Berthold Koletzko is a recipient of the Freedom to Discover Award of the Bristol-Myers-Squibb Foundation, New York, New York, USA. The studies reported herein have been carried out with the partial financial support from the Commission of the European Communities, within the 7th Framework Programme, EARLY NUTRITION, KBBE.2011.2.2-036. None of the authors are affiliated to any advisory board or have financial or personal interest in any organization sponsoring the research.

\section{REFERENCES AND RECOMIMENDED}

\section{READING}

Papers of particular interest, published within the annual period of review, have been highlighted as:

- of special interest

- of outstanding interest

Additional references related to this topic can also be found in the Current

World Literature section in this issue (pp. 360-361).

1. Staat BC, Galan HL, Harwwod JE, et al. Transplacental supply of mannose and inositol in uncomplicated pregnancies using stable isotopes. J Clin Endocrinol Metab 2012; 97:2497-2502

2. Illsley NP. Glucose transporters in human placenta. Placenta 2000; 21:1420.

3. Gao L, Lv C, Xu C, et al. Differential regulation of glucose transporters mediated by $\mathrm{CRH}$ receptor type 1 and type 2 in human placental trophoblasts. Endocrinology 2012; 153:1464-1471.

4. Brown $\mathrm{K}$, Heller DS, Zamudio E, et al. Glucose transporter 3 (GLUT3) protein expression in human placenta across gestation. Placenta 2011; 32:10411049.

5. Ganguly A, McKnight RA, Raychaudhuri S, et al. Glucose transporter isoform3 mutations cause early pregnancy loss and fetal growth restriction. Am J Physiol Endocrinol Metab 2007; 292:E1241-E1255.

6. Bibee KP, Illsley NP, Moley KH. Asymmetric syncytial expression of GLUT9 splice variants in human term placenta and alterations in diabetic pregnancies. Reprod Sci 2011; 18:20-27.

7. Novakovic B, Gordon L, Robinson WP, et al. Glucose as a fetal nutrient: dynamic regulation of several glucose transporter genes by DNA methylation in the human placenta across gestation. J Nutr Biochem 2013; 24:282288.

8. Norris AW, Wang C, Yao J, et al. Effect of insulin and dexamethasone on fetal assimilation of maternal glucose. Endocrinology 2011; 152:255262.

9. Jansson N, Rosario FJ, Gaccioli F, et al. Activation of placental mTOR

- signaling and amino acid transporters in obese women giving birth to large babies. J Clin Endocrinol Metab 2013; 98:105-113.

Influence of maternal obesity on placental mTOR signaling.

10. Jansson T, Aye IL, Goberdhan DC. The emerging role of mTORC1 signaling in

- placental nutrient-sensing. Placenta 2012; 33 (Suppl. 2):e23-e29.

An interesting review on mTOR as nutrient sensor in placenta.

11. Liu $B, X u Y$, Voss $C$, et al. Altered protein expression in gestational diabetes mellitus placentas provides insight into insulin resistance and coagulation/ fibrinolysis pathways. PLoS One 2012; 7:e44701. doi: 10.1371/journal. pone.0044701

12. Leonce J, Brockton $\mathrm{N}$, Robinson $\mathrm{S}$, et al. Glucose production in the human placenta. Placenta 2006; 27:103-108.

13. Schaefer-Graf UM, Graf K, Kulbacka I, et al. Maternal lipids as strong determinants of fetal environment and growth in pregnancies with gestational diabetes mellitus. Diabetes Care 2008; 31:1858-1863.

14. Gil-Sanchez A, Larque $E$, Demmelmair $H$, et al. Maternal-fetal in vivo transfer of $\left[{ }^{13} \mathrm{C}\right]$ docosahexaenoic and other fatty acids across the human placenta $12 \mathrm{~h}$ after maternal oral intake. Am J Clin Nutr 2010; 92:115-122.

15. Larque $\mathrm{E}$, Demmelmair $\mathrm{H}$, Gil-Sánchez $A$, et al. Placental transfer of fatty acids and fetal implications. Am J Clin Nutr 2011; 94:1908S-1913S

16. Lattka $E$, Koletzko B, Zeilinger $S$, et al. Umbilical cord PUFA are determined by maternal and child fatty acid desaturase (FADS) genetic variants in the Avon Longitudinal Study of Parents and Children (ALSPAC). Br J Nutr 2012; 9: $1-15$.
17. Gil-Sanchez A, Koletzko B, Larque E. Current understanding of placental fatty - acid transport. Curr Opin Clin Nutr Metab Care 2012; 15:265-272.

A recent review on the mechanisms involved in the placental fatty acid transfer.

18. Duttaroy AK. Transport of fatty acids across the human placenta: a review. Prog Lipid Res 2009; 48:52-61.

19. Bildirici I, Roh CR, Schaiff WT, et al. The lipid droplet-associated protein adipophilin is expressed in human trophoblasts and is regulated by peroxisomal proliferator-activated receptor-gamma/retinoid $\mathrm{X}$ receptor. J Clin Endocrinol Metab 2003; 88:6056-6062.

20. Magnusson $\mathrm{AL}$, Waterman IJ, Wennergren $\mathrm{M}$, et al. Triglyceride hydrolase activities and expression of fatty acid binding proteins in the human placenta in pregnancies complicated by intrauterine growth restriction and diabetes. J Clin Endocrinol Metab 2004; 89:4607-4614.

21. Gauster $M$, Hiden $U$, van Poppel $M$, et al. Dysregulation of placental endothelial lipase in obese women with gestational diabetes mellitus. Diabetes 2011; 60:2457-2464.

22. Dubé E, Gravel A, Martin C, et al. Modulation of fatty acid transport and

- metabolism by maternal obesity in the human full-term placenta. Biol Reprod 2012; 87:14; 1-11.

Influence of maternal obesity on placental fatty acid carriers.

23. Radaelli $T$, Lepercq J, Varastehpour $A$, et al. Differential regulation of genes for fetoplacental lipid pathways in pregnancy with gestational and type 1 diabetes mellitus. Am J Obstet Gynecol 2009; 201:209.e1-209.e210.

24. Thomas $B$, Ghebremskel $K$, Lowy $C$, et al. Plasma $A A$ and $D H A$ levels are not compromised in newly diagnosed gestational diabetic women. Eur J Clin Nutr 2004; 58:1492-1497.

25. Wijendran V, Bendel RB, Couch SC, et al. Fetal erythrocyte phospholipid polyunsaturated fatty acids are altered in pregnancy complicated with gestational diabetes mellitus. Lipids 2000; 35:927-931.

26. Pitkin RM, Connor WE, Lin DS. Cholesterol metabolism and placental transfer in the pregnant Rhesus monkey. J Clin Invest 1972; 51:2584-2592.

27. Napoli C, DÁrmiento FP, Mancini FP, et al. Fatty streak formation occurs in human fetal aortas and is greatly enhanced by maternal hypercholesterolemia. Intimal accumulation of low density lipoprotein and its oxidation precede monocyte recruitment into early atherosclerotic lesions. J Clin Invest 1997; 100:2680-2690.

28. Woollett LA. Review: Transport of maternal cholesterol to the fetal circulation.

- Placenta 2011; 32 (Suppl. 2):S218-S221.

An interesting review on the mechanism involved in the cholesterol placental transfer.

29. Lindegaard $M L$, Wassif $C A$, Vaisman $B$, et al. Characterization of placental cholesterol transport: ABCA1 is a potential target for in utero therapy of Smith-Lemli-Opitz syndrome. Hum Mol Genet 2008; 17:3806-3813.

30. Nikitina $L$, Wenger $F$, Baumann $M$, et al. Expression and localization pattern of ABCA1 in diverse human placental primary cells and tissues. Placenta 2011; 32:420-430.

31. Aye IL, Wadell BJ, Mark PJ, et al. Placental ABCA1 and ABCG1 transporters efflux cholesterol and protect trophoblasts from oxysterol induced toxicity. Biochim Biophys Acta 2010; 1801:1013-1024.

32. Bhattacharjee J, letta F, Giacomello E, et al. Expression and localization of ATP binding cassette transporter A1 (ABCA1) in first trimester and term human placenta. Placenta 2010; 31:423-430.

33. Smilde-Baardman ME, Kerstjens-Frederikse WS, Berger RM, et al. The role of maternal-fetal cholesterol transport in early fetal life: current insights. Biol Reprod 2012; 14: doi: 10.1095/biolreprod.112.102442

34. Plosch $T$, Gellhaus $A$, van Straten EM, et al. The liver $X$ receptor (LXR) and its target gene ABCA1 are regulated upon low oxygen in human trophoblast cells: a reason for alterations in preeclampsia? Placenta 2010; 31:910-918.

35. Wadsack C, Tabano S, Maier A, et al. Intrauterine growth restriction is associated with alterations in placental lipoprotein receptors and maternal lipoprotein composition. Am J Physiol Endocrinol Metab 2007; 292:E476E484.

36. Avagliano L, Garo C, Marconi AM. Placental amino acids transport in intrauterine growth restriction. J Pregnancy 2012; 11: doi: 10.1155/2012/ 972562

37. Cleal JK, Lewis RM. The mechanisms and regulation of placental amino acid transport to the human fetus. J Neuroendocrinol 2008; 20:419-426.

38. Cleal JK, Glazier JD, Ntani G, et al. Facilitated transporters mediate net efflux of amino acids to the fetus across the basal membrane of the placental syncytiotrophoblast. J Physiol 2011; 589:987-997.

39. Roos $\mathrm{S}$, Lagerlof $\mathrm{O}$, Wenergren $\mathrm{M}$, et al. Regulation of placental amino acid transporter activity by mammalian target of rapamycin. Am J Physiol Cell Physiol 2009; 296:C142-C150.

40. Roos S, Jansson N, Palmberg I, et al. Mammalian target of rapamycin in the human placenta regulates leucine transport and is down-regulated in restricted foetal growth. J Physiol 2007; 582:449-459.

41. Rosario FJ, Schumacher MA, Jiang J, et al. Chronic maternal infusion of full-

length adiponectin in pregnant mice down-regulates placental amino acid transporter activity and expression and decreases fetal growth. J Physiol 2012; 590:1495-1509.

A new mechanism of lipid metabolism with mTOR nutrient signal in placenta.

42. Tsitsiou E, Sibley CP, D'Souza SW, et al. Homocysteine transport by systems $\mathrm{L}, \mathrm{A}$ and $\mathrm{y}+\mathrm{L}$ across the microvillous plasma membrane of human placenta. J Physiol 2009; 587:4001-4013. 